\title{
Stochastic thermodynamics of fluctuating density fields: Non-equilibrium free energy differences under coarse-graining
}

\author{
T. Leonard, ${ }^{1}$ B. Lander, ${ }^{1}$ U. Seifert, ${ }^{1}$ and T. Speck ${ }^{2}$ \\ ${ }^{1}$ II. Institut für Theoretische Physik, Universität Stuttgart, Pfaffenwaldring 57, 70550 Stuttgart, Germany \\ ${ }^{2}$ Institut für Theoretische Physik II, Heinrich-Heine-Universität Düsseldorf, \\ Universitätsstraße 1, 40225 Düsseldorf, Germany
}

\begin{abstract}
We discuss the stochastic thermodynamics of systems that are described by a time-dependent density field, for example simple liquids and colloidal suspensions. For a time-dependent change of external parameters, we show that the Jarzynski relation connecting work with the change of free energy holds if the time evolution of the density follows the Kawasaki-Dean equation. Specifically, we study the work distributions for the compression and expansion of a two-dimensional colloidal model suspension implementing a practical coarse-graining scheme of the microscopic particle positions. We demonstrate that even if coarse-grained dynamics and density functional do not match, the fluctuation relations for the work still hold albeit for a different, apparent, change of free energy.
\end{abstract}

PACS numbers: 05.40.-a,05.70.Ln

\section{INTRODUCTION}

The estimation of absolute and relative free energies lies at the very heart of computational chemistry. Accordingly, a wide range of methods such as thermodynamic integration and umbrella sampling has been developed, see, e.g., Refs. 13 for recent reviews. With the advent of fluctuation theorems [4-7] and the Jarzynski relation [8], non-equilibrium methods have been added to this arsenal. These non-equilibrium methods promise to extract free energy differences from switching trajectories that are harvested out of equilibrium. It has been argued that non-equilibrium methods generally are less efficient than conventional methods 9 . There might be, however, situations where they constitute the only possibility, e.g. in force-probe experiments and molecular dynamics simulations of large biomolecules [10]. Moreover, the free energy estimated from trajectories generated under uni-directional protocols is biased, a problem that can be alleviated by bi-directional sampling schemes employing Bennett's acceptance ratio method [11 13].

An open question in stochastic thermodynamics [14] is how coarse-graining and hidden degrees of freedom influence the statistics, and therefore the fluctuation theorems, of work and heat 15,22 . For example, overdamped dynamics is typically used to model soft matter systems on time scales larger than the relaxation time of momenta. In this case a reduced description is found by eliminating fast degrees of freedom. However, in an environment with a spatially varying temperature there is a contribution to the entropy production (due to microscopic cycles) that is missed by the overdamped dynamics 21]. Another possibility is to eliminate slow degrees of freedom: In Ref. 20 the position of a colloidal particles has been measured that is coupled to another "hidden" particle, leading to a modified slope of the fluctuation theorem. For discrete state spaces, coarse-graining based on a time-scale separation has been discussed for removing fast states [16 18] and the clustering of microstates into mesostates [15, 19].

In this paper, we consider another kind of coarsegraining arising in many-body systems, where we might no longer be able (or interested) to fully resolve the microscopic particle positions. Instead, we measure a coarse-grained density profile by counting particles in a probe volume over some time. This density fluctuates, i.e., repeating the experiment we will obtain slightly different densities for each realization. Such a behavior calls for a field theoretical treatment. In Sec. II], we make the link to stochastic thermodynamics by proving the Jarzynski relation under two conditions: (i) The time evolution of density fluctuations are governed by the KawasakiDean equation [23, 24] and (ii) the free energy functional that enters the Kawasaki-Dean equation is given by the same functional that determines the equilibrium weight of density fluctuations away from the most probable density profile. There is a close link to density functional theory [25 27, which has been applied successfully to study the structural and thermodynamics properties of a wide range of spatially inhomogeneous systems. As an illustration, in Sec. III we consider the compression and expansion of a colloidal suspension. While this driving protocol has been studied previously [28, 31, here we employ it to investigate the effects of coarse-graining on the work distribution and the resulting change of free energy.

\section{STOCHASTIC THERMODYNAMICS OF COARSE-GRAINED DENSITIES}

\section{A. Coarse-grained densities}

We consider a system of particles confined in a volume $V$ in contact with a heat reservoir at temperature $T$ (throughout we set Boltzmann's constant to unity). For simplicity, we restrict ourselves to a constant number of particles $N$ but the extension to a particle reservoir is straightforward. We assume that the density $\rho(\mathbf{r})$ 
is the result of some coarse-graining procedure in space, in time, or both, of the microscopic degrees of freedom. Hence, $\rho(\mathbf{r})$ fluctuates, and fluctuations away from the most probable profile are governed by the Boltzmann distribution

$$
\psi[\rho]=e^{-(F[\rho]-\Phi) / T}
$$

with free energy functional $F[\rho]$. This functional follows from the definition of the Helmholtz free energy $\Phi$ through

$$
e^{-\Phi / T} \equiv \int \mathrm{d} \mathbf{r}_{1} \cdots \mathrm{d} \mathbf{r}_{N} e^{-\hat{U} / T}=\int[\mathrm{d} \rho] e^{-F[\rho] / T}
$$

as

$$
F[\rho]=-T \ln \int \mathrm{d} \mathbf{r}_{1} \cdots \mathrm{d} \mathbf{r}_{N} \delta\left[\rho(\mathbf{r})-\rho_{\mathrm{CG}}(\mathbf{r})\right] e^{-\hat{U} / T},
$$

where $\hat{U}\left(\left\{\mathbf{r}_{k}\right\}\right)$ is the potential energy and $\rho_{\mathrm{CG}}\left(\mathbf{r} \mid\left\{\mathbf{r}_{k}\right\}\right)$ formalizes the coarse-graining procedure through mapping a set of coordinates onto a density field. The free energy functional $F[\rho]=F_{\text {id }}[\rho]+U[\rho]$ of a given density profile $\rho(\mathbf{r})$ is customarily split into an ideal gas part

$$
F_{\text {id }}[\rho] \equiv T \int \mathrm{d} \mathbf{r} \rho(\mathbf{r})[\ln \rho(\mathbf{r})-1]
$$

and the excess free energy $U[\rho]$, which is not known explicitly in general.

In order to proceed, we need to provide an equation of motion that governs the temporal evolution of the density field $\rho(\mathbf{r}, t)$. This has been a point of some debate as reviewed by Archer and Rauscher [32. Let us first consider the microscopic density

$$
\hat{\rho}(\mathbf{r}, t) \equiv \sum_{k=1}^{N} \delta\left(\mathbf{r}-\mathbf{r}_{k}(t)\right)
$$

where $\mathbf{r}_{k}$ denotes the position of the $k$ th particle. Here, no coarse-graining has been performed and we assume that we have full knowledge of all particle positions. In this case the excess part of the density functional equals the potential energy, $U[\hat{\rho}]=\hat{U}=\sum_{k<l} u\left(\left|\mathbf{r}_{k}-\mathbf{r}_{l}\right|\right)$ (assuming pairwise interactions), and can thus be expressed through the quadratic form

$$
U[\rho] \equiv \frac{1}{2} \int \mathrm{d} \mathbf{r} \mathrm{d} \mathbf{r}^{\prime} \rho(\mathbf{r}) u\left(\left|\mathbf{r}-\mathbf{r}^{\prime}\right|\right) \rho\left(\mathbf{r}^{\prime}\right) .
$$

Starting from the overdamped stochastic motion of $N$ particles interacting via the pair potential $u(r)$, Dean [24] has shown that the microscopic density Eq. (5) exactly obeys the equation of motion

$$
\partial_{t} \hat{\rho}=\nabla \cdot\left[\hat{\rho} \nabla \frac{\delta F[\hat{\rho}]}{\delta \hat{\rho}}+\boldsymbol{\xi}\right]
$$

where the noise has zero mean and correlations

$$
\left\langle\xi_{i}(\mathbf{r}, t) \xi_{j}\left(\mathbf{r}^{\prime}, t^{\prime}\right)\right\rangle=2 T \hat{\rho}(\mathbf{r}, t) \delta_{i j} \delta\left(\mathbf{r}-\mathbf{r}^{\prime}\right) \delta\left(t-t^{\prime}\right) .
$$

The noise is thus multiplicative and vanishes in regions where the density is zero, i.e., particles are absent.

It is instructive to emphasize the difference to classical density functional theory (DFT) 33, which is based on the functional

$$
\mathcal{F}[\rho] \equiv \min _{\Psi \mid \rho} \int \mathrm{d} \mathbf{r}_{1} \cdots \mathrm{d} \mathbf{r}_{N} \Psi[\hat{U}+T \ln \Psi],
$$

where $\Psi\left(\left\{\mathbf{r}_{k}\right\}\right)$ is the full many-body distribution. This functional is obtained as a constrained minimization over all normalized distributions $\Psi$ that yield the density profile $\rho(\mathbf{r})=\int \mathrm{d} \mathbf{r}_{1} \cdots \mathrm{d} \mathbf{r}_{N} \Psi \hat{\rho}$. In particular, the equilibrium density $\langle\hat{\rho}\rangle_{0}$ fulfills

$$
\left.\frac{\delta \mathcal{F}[\rho]}{\delta \rho}\right|_{\langle\hat{\rho}\rangle_{0}}=\mu
$$

where $\mu$ is the chemical potential. The free energy functional $\mathcal{F}[\rho]$ can again be split into the ideal part Eq. (4) and an excess part. The latter is not known in general, however, excellent approximations have been obtained for, e.g., hard spheres (see Ref. 27 and references therein). Since densities in DFT are ensemble averages, dynamical DFT results in a deterministic equation

$$
\partial_{t} \rho=\nabla \cdot\left[\rho \nabla \frac{\delta \mathcal{F}[\rho]}{\delta \rho}\right]
$$

lacking the noise term from Eq. (7). Note that Eq. (11) follows from an adiabatic approximation assuming that the two-body density in the time-dependent case is that of the stationary equilibrium system at the same onebody density $\rho(\mathbf{r})$. Non-adiabatic corrections have been discussed recently within a variational approach based on Rayleigh's dissipation functional [34].

Here, we are interested in the case of fluctuating densities $\rho(\mathbf{r}, t)$ that are the result of a coarse-graining procedure. We assume that Eq. (7) in conjunction with the correct free energy functional, which we will refer to as the Kawasaki-Dean equation, holds for the temporal evolution of such a coarse-grained density profile. For the microscopic density $\hat{\rho}$ using Eq. (6), this is an exact result. Note that the free energy functional $F[\rho]$, in general, is different from $\mathcal{F}[\rho]$ and does depend on the details of the coarse-graining procedure, i.e., the excess part will be different from the quadratic form Eq. (6).

\section{B. Stochastic thermodynamics}

Suppose that we drive the system out of thermal equilibrium by changing one or more external parameters, which we denote $\lambda$. The total change of the free energy functional

$$
\frac{\mathrm{d} F[\rho]}{\mathrm{d} t}=\int \mathrm{d} \mathbf{r} \frac{\delta F[\rho]}{\delta \rho(\mathbf{r}, t)} \partial_{t} \rho(\mathbf{r}, t)+\frac{\partial F[\rho]}{\partial \lambda} \dot{\lambda} \equiv \dot{q}_{0}+\dot{w}
$$

can be split into two terms, from which we identify the second term as the work rate $\dot{w}$. The heat $\dot{q}=\dot{q}_{0}+T \frac{\mathrm{d}}{\mathrm{d} t} S$ 
dissipated into the reservoir stems from two sources: from the change of the free energy functional and from the change of the constrained intrinsic entropy $S[\rho]$ associated with the set of different microstates corresponding to the density profile $\rho$, see Ref. 14 for a detailed discussion. We introduce trajectories as histories of density profiles in time, $\Gamma \equiv\{\rho(\mathbf{r}, t): 0 \leqslant t \leqslant \tau\}$, and a protocol $\lambda(t)$ that describes the switching between initial, $\lambda(0)=\lambda_{0}$, and final, $\lambda(\tau)=\lambda_{1}$, state.

The central quantity that enters the derivation of fluctuation theorems is the ratio of the probabilities $\mathcal{P}$ for forward and backward trajectories,

$$
\mathcal{R}[\Gamma ; \lambda] \equiv \ln \frac{\mathcal{P}[\Gamma ; \lambda]}{\mathcal{P}\left[\Gamma^{\dagger} ; \lambda^{\dagger}\right]},
$$

where $\Gamma^{\dagger}=\{\rho(\mathbf{r}, \tau-t): 0 \leqslant t \leqslant \tau\}$ and $\lambda^{\dagger}(t)=\lambda(\tau-t)$ denote the time reversal of trajectory and protocol, respectively. The weight $\mathcal{P}[\Gamma ; \lambda]$ of a single trajectory depends on the dynamics of the density fluctuations. Assuming that the temporal evolution Eq. (7) still holds for a coarse-grained density $\rho(\mathbf{r})$ with the appropriate free energy functional $F[\rho]$, we show in appendix A that the ratio Eq. (13) becomes

$$
\mathcal{R}=-\frac{q_{0}}{T}+\ln \frac{\psi_{0}[\rho(\mathbf{r}, 0)]}{\psi_{1}[\rho(\mathbf{r}, \tau)]},
$$

where

$$
q_{0} \equiv \int_{0}^{\tau} \mathrm{d} t \dot{q}_{0}=\int \mathrm{d} t \mathrm{~d} \mathbf{r} \frac{\delta F[\rho]}{\delta \rho(\mathbf{r}, t)} \partial_{t} \rho(\mathbf{r}, t) .
$$

The boundary term is given by the equilibrium Boltzmann distributions $\psi_{\lambda}$, Eq. (1), of initial and final state.

Equipped with the ratio Eq. (14), a number of results can be obtained following the standard approach 14 . The arguably most prominent is the Jarzynski relation 8

$$
\left\langle e^{-w / T}\right\rangle=\int[\mathrm{d} \Gamma] e^{-w / T} \mathcal{P}=e^{-\Delta \Phi / T},
$$

which follows through combining Eqs. (13), 14), and (1) with $F_{1}-F_{0}=q_{0}+w$ [Eq. (12)]. Eq. (16) relates the average over non-equilibrium trajectories on the left hand side with the change $\Delta \Phi \equiv \Phi_{1}-\Phi_{0}$ of the equilibrium Helmholtz free energy Eq. (2) between final and initial state on the right hand side.

The most common approach to calculate the free energy difference $\Delta \Phi$ between two states is thermodynamic integration 35, which corresponds to a quasi-static process $(\dot{\lambda} \rightarrow 0)$. In practice, one performs many equilibrium simulations at slightly different values of $\lambda$ and integrates the mean energy along this path from initial to final $\lambda$. Using Eq. (16), one could also calculate the free energy difference from trajectories at finite switching speed $\dot{\lambda} \neq 0$. There is a severe caveat one encounters trying to implement such a protocol: For a finite number $N_{\mathrm{s}}$ of trajectories, the estimator of the average

$$
\left\langle e^{-w / T}\right\rangle \simeq \frac{1}{N_{\mathrm{s}}} \sum_{n=1}^{N_{\mathrm{s}}} e^{-w_{n} / T}
$$

is dominated by rare events having a large weight due to the exponential, where $w_{n}$ is the work along the $n$th trajectory. Two ways out are to either use approximations for the work distribution or to employ bi-directional sampling. For finite but slow driving speeds $\dot{\lambda}$ the work distribution approaches a Gaussian 36, 37. but extreme tails, dominating the average Eq. (16), might be nonGaussian 38, 39. Bi-directional sampling uses work values obtained for both the forward and the reverse protocol. The free energy difference is calculated through solving

$$
\Delta \Phi=-T \ln \frac{\sum_{n}\left[1+e^{\left(w_{n}-\Delta \Phi\right) / T}\right]^{-1}}{\sum_{n}\left[e^{w_{n}^{\dagger} / T}+e^{-\Delta \Phi / T}\right]^{-1}}
$$

iteratively. Here, $w_{n}$ is the work along the $n$th trajectory under the forward protocol and $w_{n}^{\dagger}$ is the work along the $n$th trajectory under the reverse protocol. For simplicity we have assumed an equal number of trajectories for each protocol. In practice, for this procedure to converge, the work distributions for the two protocols need to overlap. Hence, even though in principle Eq. (16) is valid for any driving protocol, in practice both the approximated distribution and the bi-directional sampling require a sufficiently slow driving speed.

\section{Compression and expansion}

We drive the suspension by compressing and expanding the occupied volume $V=\lambda^{d}$ at constant particle number $N$, where $d$ is the number of dimensions. These two protocols connect two state points in the phase diagram with equal temperatures. The control parameter $\lambda$ now denotes the edge length of the volume. To calculate the work rate, we rescale lengths $\mathbf{r} \mapsto \lambda \mathbf{R}$ with

$$
\begin{aligned}
F[\rho]=T & \int \mathrm{d} \mathbf{R} \rho(\mathbf{R})\left[\ln \rho(\mathbf{R}) / \lambda^{d}-1\right] \\
& +\frac{1}{2} \int \mathrm{d} \mathbf{R} \mathrm{d} \mathbf{R}^{\prime} \rho(\mathbf{R}) u\left(\lambda\left|\mathbf{R}-\mathbf{R}^{\prime}\right|\right) \rho\left(\mathbf{R}^{\prime}\right)
\end{aligned}
$$

leading to

$$
\dot{w}[\rho]=\frac{\partial F[\rho]}{\partial \lambda} \dot{\lambda}=-P[\rho] \dot{V} .
$$

The incremental work thus has the expected form of a pressure times the volume change. The pressure is a fluctuating observable since we control the volume.

In order to obtain a concrete expression for the pressure, we employ the quadratic form Eq. (6) for the excess part of the density functional $F[\rho]$. After we have restored the original lengths, the pressure reads

$$
P[\rho] \equiv \frac{T N}{V}+\frac{1}{2 d V} \int \mathrm{d} \mathbf{r} \mathrm{d} \mathbf{r}^{\prime} \rho(\mathbf{r}, t) f\left(\left|\mathbf{r}-\mathbf{r}^{\prime}\right|\right) \rho\left(\mathbf{r}^{\prime}, t\right)
$$

with $f(r) \equiv-r u^{\prime}(r)$, where the prime denotes the derivative with respect to the argument. The first term stems 
from the ideal gas part, Eq. (4), of the free energy. The second part incorporates the interactions between particles. Inserting the microscopic density Eq. (5), it reduces to the usual microscopic expression for the virial.

\section{COLLOIDAL SUSPENSION}

\section{A. Simulation details}

As a specific illustration, we consider a model suspension of $N=400$ colloidal particles moving in two dimensions. The particles interact via the Yukawa potential

$$
u(r)=\varepsilon \frac{e^{-\kappa r}}{r}
$$

with screening length $\kappa^{-1}$ and strength of the potential $\varepsilon$, which is set to $\varepsilon=1$. Eq. 22) follows from DLVO theory [40] and is a common model for chargestabilized colloidal particles. Throughout this section, we report all energies in units of the thermal energy. Moreover, as units of length and time we use $\kappa^{-1}$ and $\left(D_{0} \kappa^{2}\right)^{-1}$, respectively, where $D_{0}$ is the short-time diffusion coefficient. We employ Brownian dynamics simulations with periodic boundaries. Particle positions are updated through integrating the coupled Langevin equations

$$
\dot{\mathbf{r}}_{k}=-\sum_{l \neq k} u^{\prime}\left(\left|\mathbf{r}_{k l}\right|\right) \frac{\mathbf{r}_{k l}}{\left|\mathbf{r}_{k l}\right|}+\boldsymbol{\xi}_{k}
$$

with time step $\Delta t=10^{-3}$, where $\mathbf{r}_{k l} \equiv \mathbf{r}_{k}-\mathbf{r}_{l}$ and the noise has correlations $\left\langle\xi_{k i}(t) \xi_{l j}\left(t^{\prime}\right)\right\rangle=2 \delta_{i j} \delta_{k l} \delta\left(t-t^{\prime}\right)$. For the force evaluation, we use a cut-off radius $r_{\mathrm{c}}=5$.

\section{B. Gaussian smearing}

For the coarse-grained density, we map the particle coordinates onto the sum of Gaussians

$$
\rho_{\mathrm{CG}}(\mathbf{r}) \equiv \sum_{k=1}^{N}\left(2 \pi \ell^{2}\right)^{-d / 2} \exp \left\{-\frac{\left|\mathbf{r}-\mathbf{r}_{k}\right|^{2}}{2 \ell^{2}}\right\},
$$

where $\ell$ is the coarse-graining length over which particle positions are smeared out. In the limit $\ell \rightarrow 0$, we recover the microscopic density $\hat{\rho}$, Eq. (5). Inserting the explicit expression Eq. 24 for the density profile into Eq. (21), we can simplify the coarse-grained pressure to a sum over particle pairs. To this end, we employ the Fourier transform of the pair potential $u(r)$ and write

$$
f(r)=-\frac{1}{(2 \pi)^{d}} \int \mathrm{d} \mathbf{q} u(q) \mathbf{q} \cdot \nabla_{\mathbf{q}} e^{\mathrm{i} \mathbf{q} \cdot \mathbf{r}} .
$$

We can now perform the integrals over the densities,

$$
\int \mathrm{d} \mathbf{r} \rho(\mathbf{r}, t) e^{\mathrm{i} \mathbf{q} \cdot \mathbf{r}}=\sum_{k=1}^{N} \exp \left\{-\frac{1}{2}(\ell q)^{2}+\mathrm{iq} \cdot \mathbf{r}_{k}\right\}
$$
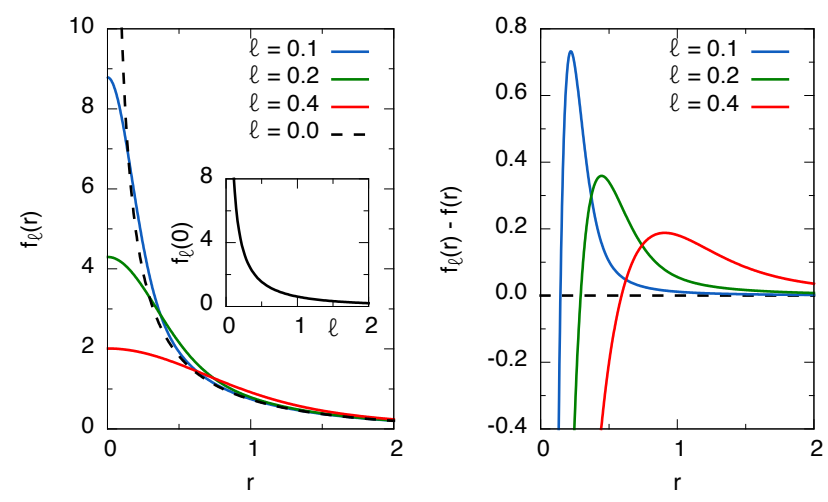

FIG. 1: (Left) The effective two-body pressure $f_{\ell}(r)$ from Eq. (28) as a function of distance $r$ for three coarse-graining lengths $\ell$ (solid lines) and the function $f(r)$ (dashed line). The inset shows the value at $r=0$ as a function of $\ell$. (Right) The difference $f_{\ell}(r)-f(r)$ is negative for small distances $r$, passes through a maximum, and then approaches zero for large distances. For larger coarse-graining length $\ell$, the distance at which zero is crossed also becomes larger.

which suggest to introduce the function $u_{\ell}(q) \equiv$ $u(q) e^{-(\ell q)^{2}}$. Performing the differentiation with respect to $\mathbf{q}$ and putting everything together, we find for the coarse-grained pressure $P[\rho(\mathbf{r}, t)]=P_{\ell}(t)$ with

$$
P_{\ell}=\frac{N}{V}+\frac{1}{d V} \sum_{k<l} f_{\ell}\left(\left|\mathbf{r}_{k}-\mathbf{r}_{l}\right|\right),
$$

where we have introduced the effective "two-body pressure"

$$
f_{\ell}(r) \equiv-r u_{\ell}^{\prime}(r)-2 \ell^{2}\left[u_{\ell}^{\prime \prime}(r)+u_{\ell}^{\prime}(r) / r\right]
$$

and $u_{\ell}(r)$ is the back-transformation of $u_{\ell}(q)$. Clearly, for $\ell=0$ we recover $f_{0}(r)=f(r)$.

The advantage of Eq. 27) is that we can calculate the coarse-grained pressure (and therefore the work) for a range of coarse-graining lengths from a single simulation integrating Eq. (23). Inserting for $d=2$ dimensions the known Fourier transform of Eq. 22, we obtain

$$
u_{\ell}(r)=\int_{0}^{\infty} \mathrm{d} q \frac{q}{\sqrt{1+q^{2}}} e^{-(\ell q)^{2}} J_{0}(q r),
$$

where $J_{0}(x)$ is the zero-order Bessel function of the first kind. Taking the derivative with respect to $r$ leads to integrals involving higher-order Bessel functions. For a predefined set of lengths $\ell$, we numerically evaluate the resulting integrals and tabulate the functions Eq. 28 required for calculating the coarse-grained pressure from a given particle configuration. In Fig. 1. we plot $f_{\ell}(r)$ and the difference $f_{\ell}(r)-f(r)$. While $f(r)$ diverges for $r \rightarrow 0$, this divergence is removed for any $\ell>0$ and the apparent pressure $f_{\ell}(0)$ between overlapping particles remains finite. The inset shows this value

$$
f_{\ell}(0)=1-\sqrt{\pi} \frac{2 \ell^{2}-1}{2 \ell} e^{\ell^{2}} \operatorname{erfc}(\ell)
$$




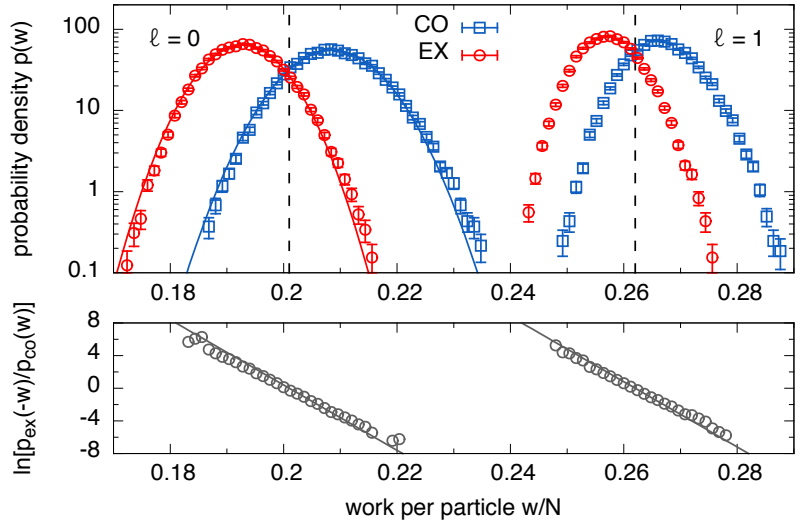

FIG. 2: (Top) Work probability distributions $p_{\text {co }}(w)$ for the compression $(\mathrm{CO})$ and $p_{\mathrm{ex}}(-w)$ for the expansion $(\mathrm{EX})$ of a two-dimensional model suspension between densities $\rho_{0}=0.1$ and $\rho_{1}=0.2$. Shown are the distributions for the microscopic density (left, $\ell=0$ ) and coarse-grained densities (right, $\ell=$ 1) using Eq. (24). The solid lines are Gaussian fits to the centers of the $\ell=0$ distributions. The vertical dashed lines indicate the free energy difference $\Delta \Phi_{\ell} / N$ calculated from Eq. 18). (Bottom) Ratio $\ln \left[p_{\text {ex }}(-w) / p_{\text {co }}(w)\right]$. The solid lines show $\Delta \Phi_{\ell}-w$ for the two values of $\ell$.

as a function of $\ell$, where $\operatorname{erfc}(x)$ denotes the complementary error function. The functions $f_{\ell}(r)$ start from a finite value but cross $f(r)$ and then approach $f(r)$ from above. Hence, there is an intermediate range in which the effective two-body pressure is increased.

\section{Work distributions}

We employ bi-directional sampling. We simulate $N_{\mathrm{s}}$ cycles during which we record one work value for the compression and one for the expansion process: During the time $\tau_{\mathrm{r}}$ the system is allowed to relax at the initial number density $\rho_{0}=N / \lambda_{0}^{2}$ and fixed $\lambda_{0}$. The system is then compressed to a higher density $\rho_{1}=N / \lambda_{1}^{2}$ during the switching time $\tau$. To this end in every time step the box length $\lambda(t)=\lambda_{0}+t\left(\lambda_{1}-\lambda_{0}\right) / \tau$ is changed linearly and all particle positions are rescaled accordingly. At the end of the compression step, the system is allowed to relax at the higher density for a time $\tau_{\mathrm{r}}$ before it is expanded during the same switching time $\tau$ to reach the initial box size, after which the cycle is repeated.

In Fig. 2, the two distributions $p_{\mathrm{co}}(w)$ and $p_{\text {ex }}(-w)$ are shown for 27000 cycles without $(\ell=0)$ and with coarsegraining (for $\ell=1$ ) for switching time $\tau=0.1$ and equilibration time $\tau_{\mathrm{r}}=4$. The centers of the distributions can be fitted well with a Gaussian, whereas the tails slightly diverge from the Gaussian shape. Under coarse-graining, the work distributions are shifted and narrower, demonstrating that the coarse-graining diminishes fluctuations. From the recorded work values for both values of $\ell$ we calculate the change of free energy $\Delta \Phi$ using Eq. (18).
For the following discussion we make the dependence of the work $w_{\ell}$ on the coarse-graining length $\ell$ explicit. The joint probabilities of actual and coarse-grained work for compression and expansion obey the fluctuation theorem 14

$$
\frac{p_{\mathrm{ex}}\left(-w_{\ell},-w_{0}\right)}{p_{\mathrm{co}}\left(w_{\ell}, w_{0}\right)}=e^{-w_{0}+\Delta \Phi} .
$$

From these joint probabilities, the Crooks work relation 41

$$
\ln \frac{p_{\mathrm{ex}}\left(-w_{0}\right)}{p_{\mathrm{co}}\left(w_{0}\right)}=\Delta \Phi-w_{0}
$$

can be derived straightforwardly. In particular, the work value $w_{*}$ where both distributions cross equals the change of free energy, $w_{*}=\Delta \Phi$, which is confirmed in Fig. 2 .

Assuming that we do not have access to the microscopic work but only to the coarse-grained work, the fluctuation theorem for the marginal probabilities becomes

$$
\frac{p_{\mathrm{ex}}\left(-w_{\ell}\right)}{p_{\mathrm{co}}\left(w_{\ell}\right)}=e^{\Delta \Phi} \int_{-\infty}^{+\infty} \mathrm{d} w_{0} p_{\mathrm{co}}\left(w_{0} \mid w_{\ell}\right) e^{-w_{0}},
$$

where $p_{\mathrm{co}}\left(w_{0} \mid w_{\ell}\right)$ is the conditional probability to observe an actual work value $w_{0}$ given that the coarse-grained work is $w_{\ell}$. At least for small $\ell$ the fluctuations of $w_{0}$ among the trajectories yielding the same $w_{\ell}$ can be expected to be sharply peaked around $w_{\ell}-\left\langle\delta w_{\ell}\right\rangle$ with differential work $\delta w_{\ell} \equiv w_{\ell}-w_{0}$. Assuming that $\delta w_{\ell}$ is independent of $w_{\ell}$, the fluctuation theorem

$$
\ln \frac{p_{\mathrm{ex}}\left(-w_{\ell}\right)}{p_{\mathrm{co}}\left(w_{\ell}\right)}=\Delta \Phi+\ln \left\langle e^{\delta w_{\ell}}\right\rangle-w_{\ell} \equiv \Delta \Phi_{\ell}-w_{\ell}
$$

for the coarse-grained work follows. In contrast to the Crooks relation for the actual work Eq. (32), now the apparent change of the Helmholtz free energy $\Delta \Phi_{\ell}$ enters. In Fig. 2 it is shown that Eq. (34) indeed holds. Note that the straight lines with slopes -1 are not fits but use the value of $\Delta \Phi_{\ell}$ calculated using Eq. (18) for the two sets of work values.

In Fig. 3 the apparent change of free energy $\Delta \Phi_{\ell}$ is plotted as a function of the coarse-graining length $\ell$ for several initial and final densities. For small $\ell$ the observed free energy increases while for large coarsegraining length it decreases again and even drops below the actual value $\Delta \Phi$. Employing the Jensen inequality $\left\langle e^{x}\right\rangle \geqslant e^{\langle x\rangle}$, we obtain the lower bound

$$
\Delta \Phi_{\ell} \geqslant \Delta \Phi+\left\langle\delta w_{\ell}\right\rangle .
$$

As shown in the inset of Fig. 3. for the system studied here this bound is already a good approximation. We can, therefore, understand the non-monotonous dependency of $\Delta \Phi_{\ell}$ from the mean differential work and, consequently, from the behavior of $f_{\ell}(r)$ plotted in Fig. 11 For small $\ell$, the effective two-body pressure $f_{\ell}(r)>f(r)$ is increased for typical particle distances, leading to larger 


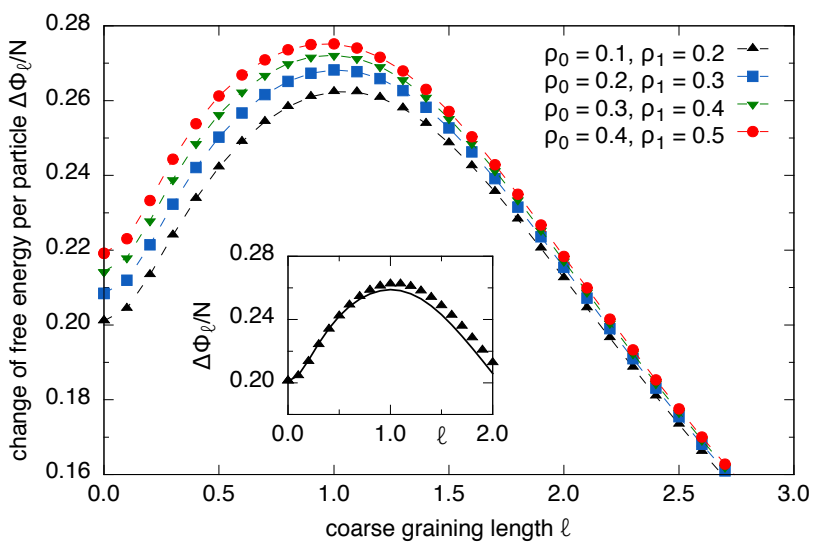

FIG. 3: (Apparent) change of free energy per particle $\Delta \Phi_{\ell} / N$ as a function of coarse-graining length $\ell$ for several low $\left(\rho_{0}\right)$ and high $\left(\rho_{1}\right)$ densities. The dashed lines are guides to the eye. The inset shows the change $\Delta \Phi_{\ell} / N$ (symbols) together with the lower bound $\Delta \Phi / N+\left\langle\delta w_{\ell}\right\rangle / N$ (solid line).

work values. Increasing $\ell$ to be larger than the typical particle distance, the values of $f_{\ell}(r)$ sampled are typically smaller than $f(r)$ and consequently also the work values are smaller compared to the actual work.

\section{CONCLUSIONS}

To summarize, we have studied the stochastic thermodynamics of density fields $\rho(\mathbf{r})$ originating from a coarsegraining procedure of microscopic particle positions. To this end, we have assumed that the Dean equation (7), originally derived for the microscopic density, also holds for coarse-grained density fields. While there is a oneto-one mapping between particle positions and microscopic density, this is not necessarily the case for coarsegrained "smeared" density profiles, where information is lost. A more rigorous route to obtain the evolution equation would be to employ the Mori-Zwanzig projection formalism [42, 43. The Kawasaki-Dean equation is a, on this level uncontrolled, Markovian approximation that is, however, thermodynamically consistent. By this we mean that employing the constrained free energy functional $F[\rho]$ that determines the weight of equilibrium fluctuations to generate both the dynamics and the work, the Jarzynski relation Eq. (16) holds and yields the correct change of the free energy for systems driven by time-dependent protocols. Note that a more general functional form for the mobility $D[\rho]$ in Eq. (7) (instead of just $D[\rho]=\rho$ ) will not change this result as long as the noise obeys the fluctuation-dissipation theorem, i.e., the noise correlations are proportional to $D$. Such general mobilities arise in the macroscopic fluctuation theory (see Ref. 44 and references therein), which allows to calculate the large deviation functional (the "free energy") for the density profile in non-equilibrium steady states.

The functional $F[\rho]$ is not known in general. Although it is in principle different from the DFT density functional $\mathcal{F}[\rho]$, both have to be constructed approximately. Since in the limit of small fluctuations the most probable density profile minimizing $F[\rho]$ will be close to the equilibrium profile minimizing $\mathcal{F}[\rho]$, both functionals will practically be identical. Our results might thus open a route to also investigate and improve the thermodynamic consistency of density functionals and to use dynamical density functional theory not only for relaxing but also for driven dynamics.

As a first step in this direction, we have studied the compression and expansion of a two-dimensional model colloidal suspension. We construct the density field Eq. (24) as a sum of Gaussians with width $\ell$ centered at the particle positions. As approximation for the density functional, we employ the quadratic form Eq. (6). While the microscopic dynamics is governed by the pair potential $u(r)$, the work is calculated from an effective pair potential $u_{\ell}(r)$ that depends on the coarse-graining length. We employ the Yukawa potential, for which the integral Eq. (29) can be performed. For potentials with a steeper repulsion, a microscopic cut-off has to be employed. The free energy difference extracted using Eq. (18) is a nonmonotonous function of the coarse-graining length and can be rationalized from the functional form of the effective potential. Moreover, we have shown that quite general insights into the work distributions of coarse-grained processes can be obtained from joint probabilities of both microscopic and coarse-grained work. In particular, the Jarzynski relation and the Crooks relation Eq. (34) hold involving the change of an apparent free energy that depends on the coarse-graining length. This demonstrates that care has to be taken: Even if the fluctuation theorem exhibits the correct slope, the free energy change might be systematically effected by measurement uncertainties.

\section{Appendix A: Antisymmetric part of the stochastic action}

The derivation presented here follows standard arguments for Gaussian noise (see, e.g., Ref. 45]) starting with Eq. 77). In the following, it will become convenient to define the scalar noise $\zeta(\mathbf{r}, t) \equiv \nabla \cdot \boldsymbol{\xi}(\mathbf{r}, t)$ with correlations

$$
\begin{aligned}
K\left(\mathbf{r}, t \mid \mathbf{r}^{\prime}, t^{\prime}\right) & \equiv\left\langle\zeta(\mathbf{r}, t) \zeta\left(\mathbf{r}^{\prime}, t^{\prime}\right)\right\rangle \\
& =2 T \delta\left(t-t^{\prime}\right) \nabla \cdot \nabla^{\prime} \rho(\mathbf{r}, t) \delta\left(\mathbf{r}-\mathbf{r}^{\prime}\right),
\end{aligned}
$$

where $\nabla^{\prime}$ acts on $\mathbf{r}^{\prime}$. The probability of a noise history is Gaussian, $\mathcal{P}[\zeta]=e^{-\mathcal{A}[\zeta]}$, with stochastic action

$$
\mathcal{A}[\zeta] \equiv \frac{1}{2} \int \mathrm{d} \mathbf{r} \mathrm{d} t \mathrm{~d} \mathbf{r}^{\prime} \mathrm{d} t^{\prime} \zeta(\mathbf{r}, t) K^{-1}\left(\mathbf{r}, t \mid \mathbf{r}^{\prime}, t^{\prime}\right) \zeta\left(\mathbf{r}^{\prime}, t^{\prime}\right)
$$


The kernel $K^{-1}$ is the inverse of the noise correlations Eq. (A1) in the operator sense,

$$
\begin{aligned}
\int \mathrm{d} \mathbf{r}^{\prime \prime} \mathrm{d} t^{\prime \prime} K\left(\mathbf{r}, t \mid \mathbf{r}^{\prime \prime}, t^{\prime \prime}\right) K^{-1} & \left(\mathbf{r}^{\prime \prime}, t^{\prime \prime} \mid \mathbf{r}^{\prime}, t^{\prime}\right) \\
& =\delta\left(t-t^{\prime}\right) \delta\left(\mathbf{r}-\mathbf{r}^{\prime}\right) .
\end{aligned}
$$

Inserting Eq. A1 and integrating by parts, it is easy to see that the gradient of the inverse kernel can be written as

$$
\nabla K^{-1}\left(\mathbf{r}, t \mid \mathbf{r}^{\prime}, t^{\prime}\right)=\frac{1}{2 T} \frac{\delta\left(t-t^{\prime}\right)}{\rho(\mathbf{r}, t)} \nabla G\left(\mathbf{r}-\mathbf{r}^{\prime}\right)
$$

where $G\left(\mathbf{r}-\mathbf{r}^{\prime}\right)$ is the Green's function of the Laplace operator

$$
\nabla^{2} G\left(\mathbf{r}-\mathbf{r}^{\prime}\right)=-\delta\left(\mathbf{r}-\mathbf{r}^{\prime}\right)
$$

We now rearrange the evolution equation (7) for the density,

$$
\zeta(\mathbf{r}, t)=\partial_{t} \rho(\mathbf{r}, t)-\nabla \cdot \rho(\mathbf{r}, t) \nabla \frac{\delta F[\rho]}{\delta \rho(\mathbf{r}, t)}
$$

We insert this expression into Eq. A2 to obtain the stochastic action as a function of density histories. Note that changing the fields $\zeta \mapsto \rho$ implies a Jacobian. However, we are only interested in the part of the action that is antisymmetric with respect to time reversal, which reads

$$
\begin{aligned}
\mathcal{A}\left[\Gamma^{\dagger} ; \lambda^{\dagger}\right]-\mathcal{A}[\Gamma ; \lambda] & =2 \int \mathrm{d} \mathbf{r} \mathrm{d} t \mathrm{~d} \mathbf{r}^{\prime} \mathrm{d} t^{\prime}\left\{\nabla \cdot \rho(\mathbf{r}, t) \nabla \frac{\delta F[\rho]}{\delta \rho(\mathbf{r}, t)}\right\} K^{-1}\left(\mathbf{r}, t \mid \mathbf{r}^{\prime}, t^{\prime}\right) \partial_{t} \rho\left(\mathbf{r}^{\prime}, t^{\prime}\right) \\
& =-2 \int \mathrm{d} \mathbf{r} \mathrm{d} t \mathrm{~d} \mathbf{r}^{\prime} \mathrm{d} t^{\prime} \rho(\mathbf{r}, t)\left[\nabla \frac{\delta F[\rho]}{\delta \rho(\mathbf{r}, t)}\right] \cdot \nabla K^{-1}\left(\mathbf{r}, t \mid \mathbf{r}^{\prime}, t^{\prime}\right) \partial_{t} \rho\left(\mathbf{r}^{\prime}, t^{\prime}\right) \\
& =-\frac{1}{T} \int \mathrm{d} \mathbf{r} \mathrm{d} t \frac{\delta F[\rho]}{\delta \rho(\mathbf{r}, t)} \partial_{t} \rho(\mathbf{r}, t)=-\frac{q_{0}}{T},
\end{aligned}
$$

where we have used Eqs. (A4) and A5. This is the first term in Eq. (14). The full weight of a trajectory reads $\mathcal{P}[\Gamma ; \lambda]=\psi_{0}\left[\frac{1}{\rho}(\mathbf{r}, 0)\right] e^{-\mathcal{A}[\Gamma ; \lambda]}$, leading to the second term in Eq. (13) as boundary term for trajectories start- ing with density profile $\rho(\mathbf{r}, 0)$ (forward protocol) and $\rho(\mathbf{r}, \tau)$ (backward protocol). The antisymmetric part of the action is thus related to the heat dissipated into the environment as expected.
[1] F. M. Ytreberg, R. H. Swendsen, and D. M. Zuckerman, J. Chem. Phys. 125, 184114 (2006).

[2] A. Pohorille, C. Jarzynski, and C. Chipot, J. Phys. Chem. B 114, 10235 (2010).

[3] J. D. Chodera, D. L. Mobley, M. R. Shirts, R. W. Dixon, K. Branson, and V. S. Pande, Curr Opin Struct Biol. 21, 150 (2011).

[4] G. Gallavotti and E. G. D. Cohen, Phys. Rev. Lett. 74, 2694 (1995).

[5] J. Kurchan, J. Phys. A: Math. Gen. 31, 3719 (1998).

[6] J. L. Lebowitz and H. Spohn, J. Stat. Phys. 95, 333 (1999).

[7] U. Seifert, Phys. Rev. Lett. 95, 040602 (2005).

[8] C. Jarzynski, Phys. Rev. Lett. 78, 2690 (1997).

[9] H. Oberhofer, C. Dellago, and P. L. Geissler, J. Phys. Chem. B 109, 6902 (2005).

[10] R. Merkel, P. Nassoy, A. Leung, K. Ritchie, and E. Evans, Nature 397, 50 (1999).

[11] C. H. Bennett, J. Comp. Phys. 22, 245 (1976).

[12] M. R. Shirts, E. Bair, G. Hooker, and V. S. Pande, Phys.
Rev. Lett. 91, 140601 (2003)

[13] S. Kim, Y. W. Kim, P. Talkner, and J. Yi, Phys. Rev. E 86, 041130 (2012)

[14] U. Seifert, Rep. Prog. Phys. 75, 126001 (2012).

[15] S. Rahav and C. Jarzynski, J. Stat. Mech. p. P09012 (2007).

[16] A. Puglisi, S. Pigolotti, L. Rondoni, and A. Vulpiani, J. Stat. Mech. p. P05015 (2010).

[17] B. Altaner and J. Vollmer, Phys. Rev. Lett. 108, 228101 (2012).

[18] B. Altaner, S. Grosskinsky, S. Herminghaus, L. Katthän, M. Timme, and J. Vollmer, Phys. Rev. E 85, 041133 (2012).

[19] M. Esposito, Phys. Rev. E 85, 041125 (2012).

[20] J. Mehl, B. Lander, C. Bechinger, V. Blickle, and U. Seifert, Phys. Rev. Lett. 108, 220601 (2012).

[21] A. Celani, S. Bo, R. Eichhorn, and E. Aurell, Phys. Rev. Lett. 109, 260603 (2012).

[22] K. Kawaguchi and Y. Nakayama, Phys. Rev. E 88, 022147 (2013) 
[23] K. Kawasaki and T. Koga, Physica A 201, 115 (1993).

[24] D. S. Dean, J. Phys. A: Math. Gen. 29, L613 (1996).

[25] R. Evans, Adv. Phys. 28, 143 (1979).

[26] H. Löwen, Phys. Rep. 237, 249 (1994).

[27] R. Roth, J. Phys.: Condens. Matter 22, 063102 (2010).

[28] R. C. Lua and A. Y. Grosberg, J. Phys. Chem. B 109, 6805 (2005).

[29] G. E. Crooks and C. Jarzynski, Phys. Rev. E 75, 021116 (2007).

[30] S. J. Davie, J. C. Reid, and D. J. Searles, J. Chem. Phys. 136, 174111 (2012).

[31] J. Hoppenau, M. Niemann, and A. Engel, Phys. Rev. E 87, 062127 (2013).

[32] A. J. Archer and M. Rauscher, J. Phys. A: Math. Gen. 37, 9325 (2004).

[33] D. Reguera and H. Reiss, J. Chem. Phys. 120, 2558 (2004).

[34] M. Schmidt and J. M. Brader, J. Chem. Phys. 138, 214101 (2013).

[35] D. Frenkel and B. Smit, Understanding Molecular Sim- ulation: From Algorithms to Applications (Academic Press, San Diego, 2002), 2nd ed.

[36] T. Speck and U. Seifert, Phys. Rev. E 70, 066112 (2004).

[37] T. Speck, J. Phys. A: Math. Theor. 44, 305001 (2011).

[38] D. Nickelsen and A. Engel, Eur. Phys. J. B 82, 207 (2011).

[39] J. Hoppenau and A. Engel, J. Stat. Mech. 2013, P06004 (2013).

[40] J. N. Israelachvili, Intermolecular and Surface Forces (Academic Press, Amsterdam, 1992), 2nd ed.

[41] G. E. Crooks, Phys. Rev. E 60, 2721 (1999).

[42] R. Zwanzig, Phys. Rev. 124, 983 (1961).

[43] H. Grabert, Projection operator techniques in nonequilibrium statistical mechanics (Springer-Verlag, Berlin, 1982).

[44] B. Derrida, J. Stat. Mech.: Theor. Exp. 2007, P07023 (2007).

[45] T. Schmiedl, T. Speck, and U. Seifert, J. Stat. Phys. 128, 77 (2007). 